\title{
Correction to: Environmental Circadian Disruption Worsens Neurologic Impairment and Inhibits Hippocampal Neurogenesis in Adult Rats After Traumatic Brain Injury
}

\author{
Dongpeng $\mathrm{Li}^{1,2} \cdot$ Shanshan $\mathrm{Ma}^{3} \cdot$ Dewei Guo ${ }^{1} \cdot$ Tian Cheng $^{1,4} \cdot$ Hongwei $\mathrm{Li}^{1} \cdot \mathrm{Yi} \mathrm{Tian}^{1} \cdot$ Jianbin $\mathrm{Li}^{2} \cdot$ Fangxia Guan ${ }^{3}$. \\ Bo Yang ${ }^{1} \cdot$ Jian Wang ${ }^{4}$
}

Published online: 30 June 2020

(c) Springer Science+Business Media, LLC, part of Springer Nature 2020

\section{Correction to:}

Cellular and Molecular Neurobiology (2016) 36:1045-1055 https://doi.org/10.1007/s10571-015-0295-2

The original version of this article unfortunately contained an error in Fig. 4a.
The Cresyl Violet-stained image of the Sham/LL group in cortex area was given incorrectly the same as of the image in Sham/LD group, and the image of the TBI/LD group in DG area was given incorrectly the same as of the image in TBI/LL group.

Hence, the correct Fig. 4 a was given below:

The original article can be found online at https://doi.org/10.1007/ s10571-015-0295-2.

Fangxia Guan

guanfangxia@126.com

$\triangle$ Bo Yang

yangbo96@126.com

1 Department of Neurosurgery, The First Affiliated

Hospital of Zhengzhou University, Zhengzhou 450052,

People's Republic of China

2 Henan Province Red Cross Blood Center, Zhengzhou,

Henan 450014, People's Republic of China

3 School of Life Sciences, Zhengzhou University, Zhengzhou 450001, People's Republic of China

4 Department of Anesthesiology and Critical Care Medicine, Johns Hopkins University, School of Medicine, Baltimore, MD 21205, USA 

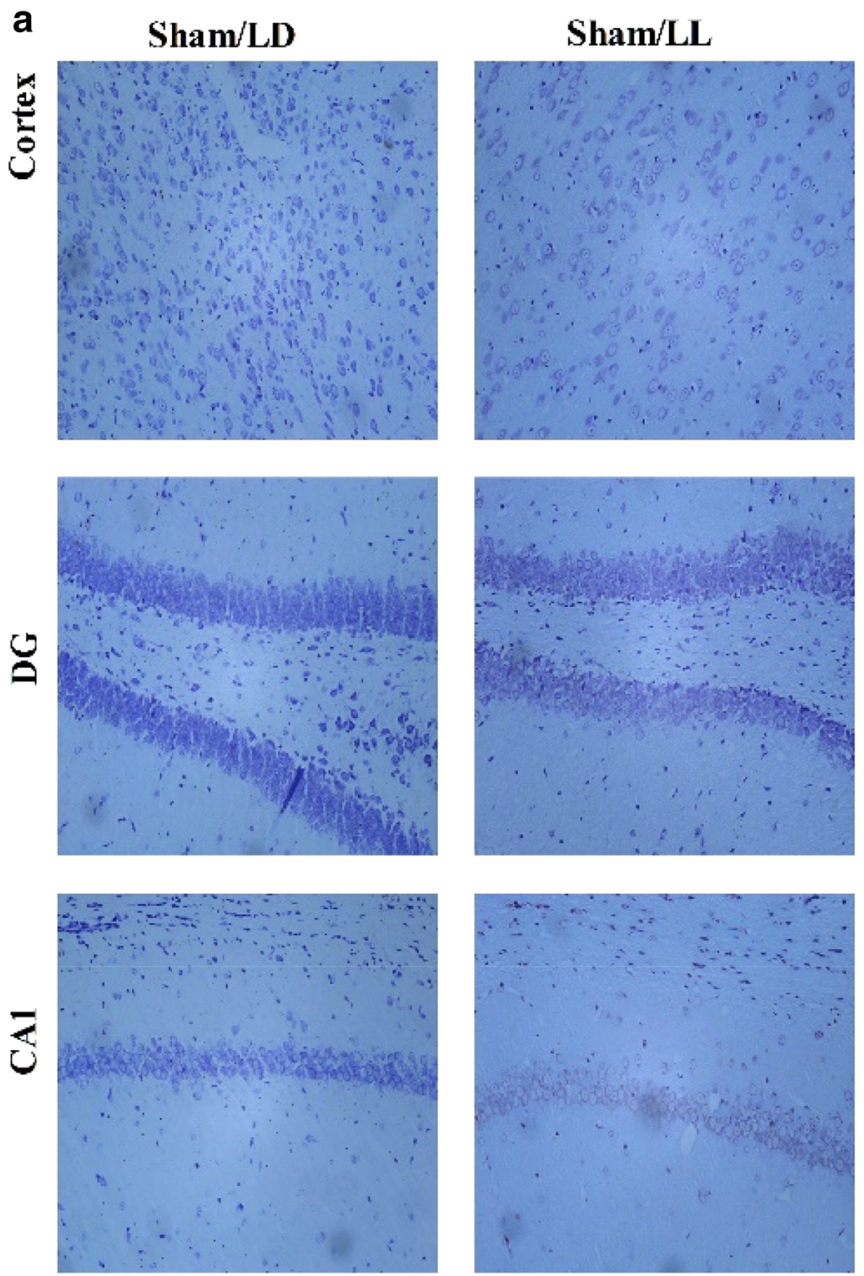

TBU/LD

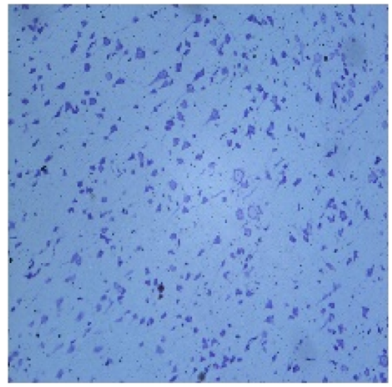

TBI/LL
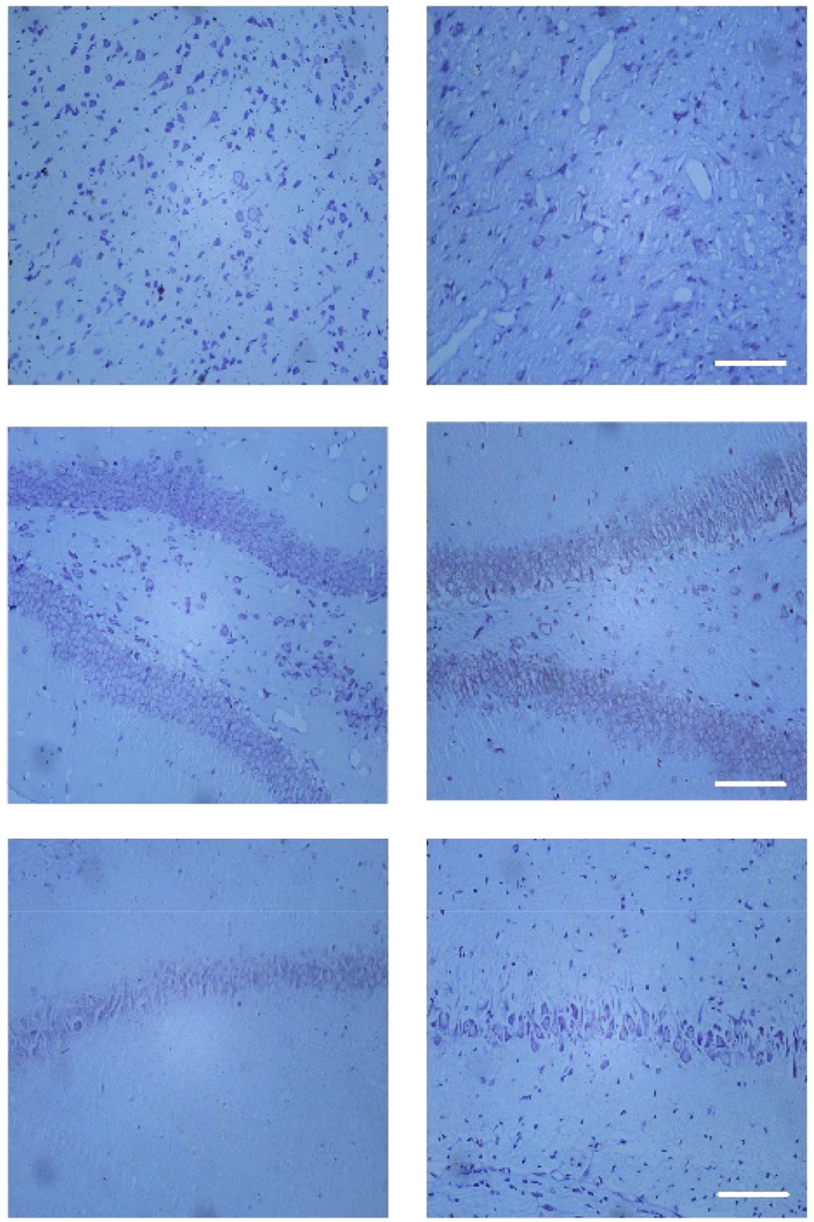

b

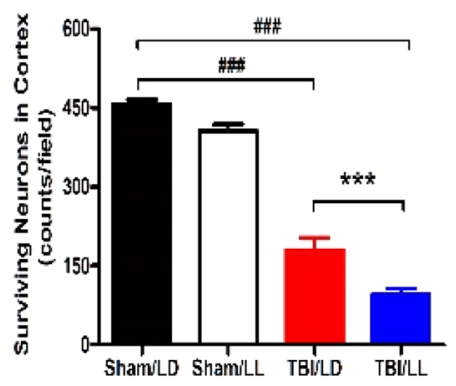

C

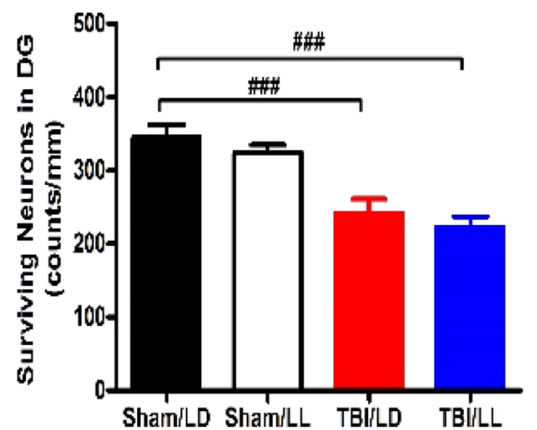

d

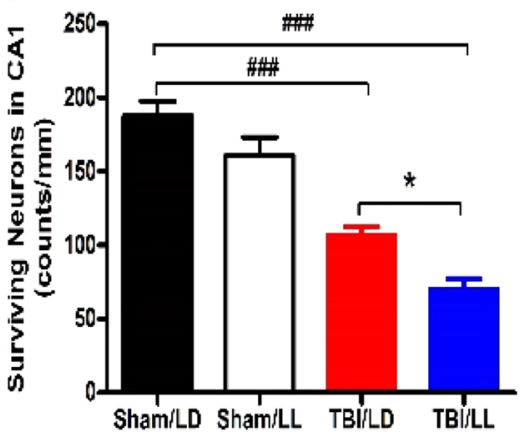

Fig. 4 Environmental circadian disruption reduces neuron survival in the cortex and hippocampus on day 14 after TBI. a Representative images of Cresyl Violet-stained brain sections. Scale bar $100 \mu \mathrm{m}$. Quantification of surviving neurons in the cortex (b), dentate gyrus
(DG; c), and hippocampal CA1 region (d). ${ }^{\#} P<0.05,{ }^{\# \#} P<0.001$ vs. Sham/LD group; $* P<0.05, * * * \mathrm{P}<0.001$ vs. TBI/LD group; one-way ANOVA followed by the Bonferroni post hoc test. Data are presented as mean $\pm \mathrm{SEM} ; n=8$ rats/group

Publisher's Note Springer Nature remains neutral with regard to jurisdictional claims in published maps and institutional affiliations. 